\title{
Polymeric micelles containing resveratrol: development, characterization, cytotoxicity on tumor cells and antimicrobial activity
}

\author{
Tamires Cunha Almeida ${ }^{\oplus *}$, Janaina Brandão Seibert², \\ Sávio Henrique de Souza Almeida ${ }^{1}$, Tatiane Roquete Amparo², \\ Luiz Fernando de Medeiros Teixeira ${ }^{3}$, Jose Mario Barichello ${ }^{4}$, \\ Bruna Bueno Postacchini', Orlando David Henrique dos Santos ${ }^{2}$, \\ Glenda Nicioli da Silva ${ }^{1}$
}

\begin{abstract}
${ }^{1}$ Laboratório de Pesquisas Clínicas, Departamento de Análises Clínicas, Universidade Federal de Ouro Preto, Campus Morro do Cruzeiro, Ouro Preto, Minas Gerais, Brazil, ${ }^{2}$ Laboratório de Fitotecnologia, Departamento de Farmácia, Universidade Federal de Ouro Preto, Campus Morro do Cruzeiro, Ouro Preto, Minas Gerais, Brazil, ${ }^{3}$ Laboratório de Microbiologia, Departamento de Análises Clínicas, Universidade Federal de Ouro Preto, Campus Morro do Cruzeiro, Ouro Preto, Minas Gerais, Brazil, ${ }^{4}$ Laboratório de Tecnologia Farmacêutica, Centro de Ciências Químicas, Farmacêuticas e de Alimentos, Universidade Federal de Pelotas, Pelotas, Campus Capão do Leão, Pelotas, Rio Grande do Sul, Brazil, ${ }^{5}$ Laboratório de Fotofísica Molecular, Departamento de Física, Universidade Federal de Ouro Preto, Campus Morro do Cruzeiro, Ouro Preto, Minas Gerais, Brazil
\end{abstract}

\begin{abstract}
Antimicrobial and antitumor activities of resveratrol, a compound found mainly in grapes, have already been demonstrated. However, its low bioavailability is a limiting factor for therapeutic application. Polymeric micelles can be an approach to solve this problem since they can encapsulate hydrophobic substances. We developed and characterized micellar formulations containing resveratrol and evaluated their cytotoxic and antimicrobial effects. The formulations were prepared by the cold dispersion method with different concentrations of F127 ( 5 or 10\% w/w) and resveratrol $(500$ or $5000 \mu \mathrm{M})$. The formulations were characterized according to size, polydispersity index, $\mathrm{pH}$, encapsulation rate and in vitro release. Cytotoxic effect was evaluated on a bladder cancer cell line and antimicrobial effect was evaluated on E. coli, S. aureus and C. albicans. One of the formulations ( $10 \% \mathrm{w} / \mathrm{w}$ of F127 and $5000 \mu \mathrm{M}$ of resveratrol) was a monodispersed solution with high encapsulation rate, thus it was chosen for the cytotoxicity and antimicrobial assays. MS$10+$ RES-3 was able to preserve the antimicrobial and cytotoxic activity of resveratrol. This is the first study that evaluated antimicrobial potential and cytotoxicity of micelles containing resveratrol on bladder cancer cells and the results showed that micellar nanostructures could ensure the maintenance of the biological activity of resveratrol.
\end{abstract}

Keywords: Antimicrobial activity. Cytotoxic activity. F127 ${ }^{\circledR}$. Polymeric micelles. Resveratrol.

*Correspondence: T. C. Almeida. Laboratório de Pesquisas Clínicas, Departamento de Análises Clínicas Universidade Federal de Ouro Preto, Campus Morro do Cruzeiro. 35400-000, Ouro Preto, Minas Gerais, Brazil. Tel.: +55 37 991533051; +55 31 35591036. E-mail: tamirescunha@hotmail.com

\section{INTRODUCTION}

Resveratrol (trans-3, 4', 5-trihydroxystilbene) is a polyphenolic compound found naturally in grapes, blueberries, raspberries and peanuts (Wang, Liu, Chen, 2013). Resveratrol has been reported to present 
antimicrobial (Lee et al., 2014; Lee et al., 2015) and antitumoral activities (Selvaraj et al., 2016; Nie et al., 2015). In addition to some in vitro studies, antimicrobial and antitumoral activities have also been demonstrated in vivo (Wu et al., 2014; Kumar, Rimando, Levenson, 2017; Euba et al., 2017) and in clinical studies (Patel et al., 2010).

For over a decade, it has been shown that resveratrol has low solubility and low bioavailability due to poor absorption and rapid metabolization (Amri et al., 2012), both physicochemical and pharmacokinetic properties that make resveratrol difficult to use clinically. Several nanoformulations with resveratrol have been developed for the treatment of tumors and infections (Santos, Veiga, Ribeiro, 2011; Figueiró et al., 2013; Friedrich et al., 2015; Vitonyte et al., 2017). Furthermore, resveratrol has some stability issues, being extremely photosensitive (Francioso et al., 2014) and unstable at alkaline $\mathrm{pH}$ and higher temperatures (Zupancic, Lavric, Kristl, 2015). However, these problems could be improved using nanoformulations and should be considered during their development.

Polymeric micelles have been studied as a drug carrier system, in particular for enhancing the solubility of hydrophobic drugs, a characteristic of many antitumor and antimicrobial agents. Some authors have shown that polymeric micelles are a suitable in vivo nanocarrier to deliver drugs with low solubility by intravenous or subcutaneous routes (Gu et al., 2017; Pellosi et al., 2016; Yu et al., 2015). Furthermore, incorporation of substances in the hydrophobic core of micelles may prevent clearance by the mononuclear phagocyte system in the spleen and liver, since the hydrophilic corona from polymeric micelles plays an important role in preventing opsonization (Rey-Rico, Cucchiarini, 2018). An in vivo pharmacokinetic study demonstrated that the area under the curve (AUC) value of resveratrol micelles was 2.73-fold higher in comparison with free resveratrol. Moreover, the mean residence time (MRT) of resveratrol micelles was significantly longer than that of resveratrol solution. The authors deduced that micelles could improve the availability of resveratrol by the slow release of drug from the micelles and evasion of clearance (Hao et al., 2017).

Micelles can be obtained by different methods, such as solvent casting, dialysis, oil/water emulsion, cold dispersion and others. The micelle preparation method is an essential factor in determining the overall micelle properties, including size, polydispersity and loading efficiency as well as its suitability for industrial scale production (Hussein, Youssry, 2018).
Pluronics $^{\circledR}$ are triblock copolymers of propylene oxide (PO) and ethylene oxide (EO) that can spontaneously self-assemble to form micelles (Kabanov, Alakhov, 2002). These copolymers are included on the US Federal Drug Administration list as safe and biocompatible compounds (Oerlemans et al., 2010). Furthermore, they are known to solubilize hydrophobic compounds in the micellar state and have been proven to be effective solubilizers of polyphenols, such as resveratrol (Wang et al., 2017). Among various types of Pluronics ${ }^{\circledR}$, F127 (F127) has gained considerable attention due to its wide range of biomedical applications (Akash, Rehman, 2015).

Therefore, micellar solutions of Pluronic ${ }^{\circledR}$ F127 containing resveratrol have been developed and characterized as a formulation for the treatment of microbial (bacterial and fungal) infection and cancer. The micellar formulation demonstrated bacteriostatic effects against $S$. aureus, E. coli and C. albicans, and furthermore demonstrated cytotoxicity on T24 bladder cancer cells. As a result, this formulation may be a good strategy for the use of resveratrol in biological systems.

\section{MATERIAL AND METHODS}

\section{Material}

Pluronic ${ }^{\circledR}$ F127, resveratrol, Dulbecco's modified Eagle's medium (DMEM), pyrene, triphenyl tetrazolium chloride, tetracycline, ketoconazole, penicillin $\mathrm{G}$ and streptomycin were purchased from Sigma-Aldrich ${ }^{\circledR}$ (St Louis, USA). Müeller-Hinton and Sabouraud medium were purchased from Himedia (Mumbai, India). Amphotericin B was purchased from Cristália (Itapira, Brazil). Methanol (HPLC grade) was purchased from Merck $^{\circledR}$ (Darmstadt, Germany). Fetal bovine serum was purchased from Cultilab Ltd. (Campinas, Brazil). Cell Proliferation Kit II (XTT) was purchased from Roche Diagnostics $^{\circledR}$ (Mannheim, Germany). Culture medium DMEM without phenol red was purchased from Invitrogen $^{\circledR}$ (Carlsbad, USA).

\section{Preparation of micellar solutions by cold dispersion method}

A weighed amount of Pluronic ${ }^{\circledR}$ F127 (5 or 10\% wt/ wt) was added to a becker containing ultrapure water and kept under moderate magnetic stirring in an ice bath $\left(4-6{ }^{\circ} \mathrm{C}\right)$ until complete dispersion of the polymer. 
The solution was kept at $10{ }^{\circ} \mathrm{C}$ for 24 hours to allow the complete dissolution of the polymer. Subsequently, the resveratrol was added and dispersed under vigorous stirring at room temperature. After preparation, all formulations were filtered on a $0.45 \mu \mathrm{m}$ filter to eliminate the non-encapsulated resveratrol (Rijcken et al., 2007). The theoretical resveratrol concentration added to the micellar solutions were 500 and $5000 \mu \mathrm{M}$. Micelles without resveratrol were also prepared and used as controls.

\section{Determination of mean size and zeta potential of the micelles}

The particle size and zeta potential values of micelles were determined with a Zetasizer (Malvern, model Zetasizer Nano series - Nano ZS) at $25^{\circ} \mathrm{C}$. The mean particle size was measured based on photocorrelation spectroscopy technique and the zeta potential was determined based on electrophoretic mobility measurements (Liu et al., 2017; Wang et al., 2017). The experiments were conducted in triplicate.

\section{Determination of $\mathrm{pH}$}

The $\mathrm{pH}$ measurement of the formulations was determined by the use of a pHmeter (Instrutemp model mPA201). The values were determined in triplicate.

\section{Quantification of resveratrol}

Resveratrol was quantified by HPLC/UV method as previously described (Trela, Waterhouse, 1996). The equipment used was the Waters e2695 coupled to a UV/Vis Waters 2485 detector. The UV/Vis detector was set at 306 $\mathrm{nm}$. Separation was done on a C18 column (Phenomenex, Luna $5 \mu, 100 \AA, 150 \times 4.6 \mathrm{~mm}$ ) at $25^{\circ} \mathrm{C}$, using methanol and ultrapure water (50:50) as mobile phase at a flow rate of $0.8 \mathrm{~mL} / \mathrm{min}$. The injection volume was $5.0 \mathrm{uL}$ and the run time for resveratrol was approximately 8 minutes. This method was used to calculated encapsulation efficiency and resveratrol in vitro release.

\section{Encapsulation efficiency}

The amount of resveratrol present on micelles was determined with HPLC/UV method previous described (Trela, Waterhouse, 1996). The formulation was filtrated on $0.45 \mu \mathrm{m}$ filter to remove non-encapsulated resveratrol and diluted on methanol/water (50:50). The encapsulation efficiency was calculated by the following equation:

Encapsulation efficiency $=\frac{\text { resveratrol concentration on micelles }}{\text { initial concentration of resveratrol }} \times 100$

\section{Micellar microenvironment analysis}

Pyrene is a fluorescent probe often used to evaluate changes in the microenvironment of a micellar system (Perry et al., 2011). Measurements of the pyrene emission spectra were conducted in methanol, water and then in methanol with different concentrations of resveratrol. Subsequently, micelles with and without resveratrol were labeled with pyrene and the emission spectra were measured. The emission spectra were measured between the wavelengths $350-500 \mathrm{~nm}$ with excitation at $335 \mathrm{~nm}$.

\section{Resveratrol in vitro release assay}

The in vitro release assay of resveratrol encapsulated at micelles was performed by dialysis membrane diffusion method at $37^{\circ} \mathrm{C}$ for $48 \mathrm{~h}$, maintaining constant agitation in a horizontally stirred incubator (Dubnoff 304D). The donor medium was composed of $1 \mathrm{~mL}$ of formulation containing about $0.5 \mu \mathrm{M}$ resveratrol, and the receptor medium was composed of phosphate buffered saline with $1 \%$ tween 80 at sink conditions (Fonseca, 2016). At pre-determined times, $1 \mathrm{~mL}$ of the receptor medium was collected and the same volume was replaced with fresh medium. The fraction collected was quantified directly, without dilution, with the HPLC/UV method previous described.

\section{Cytotoxicity on tumor cells}

The human urothelial carcinoma cell line T24 (from a high grade tumor) was purchased from the Cell Bank of the Federal University of Rio de Janeiro (Rio de Janeiro, Brazil). Cells were maintained in culture medium Dulbecco's Modified Eagle (DMEM) supplemented with $10 \%$ bovine fetal serum, $100 \mathrm{U} / \mathrm{ml}$ penicillin $\mathrm{G}, 100 \mathrm{U} / \mathrm{ml}$ streptomycin and $2,5 \mu \mathrm{g} / \mathrm{mL}$ amphotericin $\mathrm{B}$ in an atmosphere of $5 \% \mathrm{CO}_{2}$ at $37^{\circ} \mathrm{C}$.

Cytotoxicity was assessed using the Cell Proliferation Kit II (XTT, Roche ${ }^{\circledR}$ ). Briefly, $1 \times 10^{4}$ T24 cells were seeded into 96-well culture plates. After 24 hours, the cells were treated with different concentrations 
of the micellar solutions containing resveratrol $(50,100$, 150,200 and $250 \mu \mathrm{M}$ ) or with free resveratrol during 24 hours. The concentrations and the time of treatment were defined based on the study conducted by Bai et al. (2009). Cells treated with micellar solutions without resveratrol were used as controls. 24 hours after incubation, the cells were washed with Hank's solution $(0.4 \mathrm{~g} \mathrm{KCl}$, $0.06 \mathrm{~g} \mathrm{KH}_{2} \mathrm{PO}_{4}, 0.04 \mathrm{~g} \mathrm{Na}_{2} \mathrm{HPO}_{4}, 0.35 \mathrm{~g} \mathrm{NaHCO}_{3}, 1 \mathrm{~g}$ glucose and $8 \mathrm{~g} \mathrm{NaCl}$ in $1 \mathrm{~L} \cdot \mathrm{H}_{2} \mathrm{O}$ ). After washing, $12 \mathrm{uL}$ of XTT test solution ( $1 \mathrm{~mL} \mathrm{XTT} \mathrm{labeling} \mathrm{solution/20} \mathrm{uL}$ of electron-coupling reagent) were added to each well, and the absorbance was measured at 492 and $690 \mathrm{~nm}$ after 90 minutes. Absorbance results are proportional to the percentage of viable cells. Tests were conducted in triplicate. The results of absorbance were analyzed by One-way analysis of variance (ANOVA) with post hoc Tukey test using the software GraphPad Prism 6.0. A value of $p<0.05$ was considered statistically significant.

\section{Antimicrobial activity}

In vitro assays were performed using three microorganisms, representing respectively each main microbial class (gram positive and gram negative bacteria and pathogenic yeast): Staphylococcus aureus (ATCC 25923); Escherichia coli (ATCC 25922) and Candida albicans (ATCC 14408). Bacteria were cultivated in Müeller-Hinton medium by $24 \mathrm{~h}$ at $37{ }^{\circ} \mathrm{C}$ and yeast was cultivated in Sabouraud medium by $48 \mathrm{~h}$ at $37^{\circ} \mathrm{C}$. All inoculums were prepared by using direct colony suspension method in saline $(0.9 \% \mathrm{NaCl})$ with colonies selected from a $24 \mathrm{~h}$ or $48 \mathrm{~h}$ agar plate, before each assay. The suspension was adjusted to achieve a turbidity equivalent to the $0.5 \mathrm{McFarland}$ standard $(1 \mathrm{x}$ $10^{8} \mathrm{CFU} / \mathrm{mL}$ ). Antimicrobial effect of resveratrol and its formulations were evaluated by the microdilution method (CLSI, 2012). In 96-well plates, serial dilutions of 1:2 were made to obtain the same concentration (from 250 to $0.12 \mu \mathrm{M}$ ) of resveratrol for both free and formulations. The inoculums $\left(1 \times 10^{8} \mathrm{CFU} / \mathrm{mL}\right)$ were diluted 1:100 in Müeller-Hinton broth and were added in each well to obtain a final assay with $5 \times 10^{5} \mathrm{CFU} /$ mL (CLSI, 2012). For negative control only MüellerHinton broth and inoculum were added. Tetracycline $(100.0 \mu \mathrm{g} / \mathrm{mL})$ or Ketoconazole $(100.0 \mu \mathrm{g} / \mathrm{mL})$ were used as positive control for bacteria or yeast, respectively. Formulation without resveratrol was used as formulation control and the control of the broth was made without inoculum. The plates were incubated for $24 \mathrm{~h}$ or $48 \mathrm{~h}$, for bacteria or yeast, respectively at $37^{\circ} \mathrm{C}$. After the incubation, triphenyl tetrazolium chloride TTC $(0.25$ $\mathrm{mg} / \mathrm{mL}$ ) were added and the plates were again incubated for three hours. Since resveratrol is an antioxidant agent and the TTC is a redox indicator, we used a control with resveratrol and TTC (without microorganism) to ensure no interference from the compound influenced the results. The absorbance at $650 \mathrm{~nm}$ was determined in an ELISA plate reader (Molecular Devices ${ }^{\circledR}$ ). The results of absorbance were analyzed by One-way analysis of variance (ANOVA) with post hoc Tukey test using the software GraphPad Prism 6.0. A value of $p<0.05$ was considered statistically significant.

\section{RESULTS AND DISCUSSION}

Many studies have demonstrated in vitro antimicrobial and cytotoxic properties of resveratrol ( Nie et al., 2015; Selvaraj et al., 2016; Ji et al., 2015; Taylor et al., 2014; Hwang, Lim, 2015). However, there are no reports in the literature on the activity of resveratrol micellar systems against bacteria and yeast. Similarly, there are very few studies about polymeric micelles containing resveratrol for cancer treatment (Hao et al., 2017), and none about this type of formulation's effect on bladder cancer cells.

Several approaches have been investigated for Pluronic ${ }^{\circledR}$ F127 micelle preparation. Direct dissolution is a method that involves dissolving both the polymer and the drug in an aqueous solvent. Another strategy, implying the use of organic solvents, is relevant when both the copolymer and the drug are not readily soluble in water. The copolymer and the drug are dissolved in a common solvent which is then removed, usually by evaporation (Bodratti, Alexandridis, 2018). In this study, the micelles were prepared by using cold dispersion, because it is preferred when the polymeric materials are only moderately hydrophobic in nature (Bodratti, Alexandridis, 2018), as is the case with Pluronics ${ }^{\circledR}$ Furthermore there is no risk of residual organic solvent. This is an advantage of our formulation compared to that previously described by Hao et al. (2017), since those authors used a method with organic solvent.

Polymeric micelles have a critical micelle concentration (CMC) that is the lowest concentration limit for polymers to produce a micelle (Owen, Chan, Shoichet, 2012). When diluted below CMC, polymer micelles are gradually disintegrated into unimers and this can affect the solubilizing efficacy of the formulation. 
For this reason, we chose to use quantities of F127 (5 and $10 \% \mathrm{w} / \mathrm{w}$ ) that even after dilutions have concentrations higher than its CMC (2.8 $\mu \mathrm{M})$ (Stammet et al., 2010). The composition of each formulation is shown in Table I. Additionally, F127 has the characteristic of forming hydrogels at high concentrations $(>20 \% \mathrm{w} / \mathrm{w})$ (Giuliano et al., 2018), which was avoided.

The ability of the polymeric micelles to increase the aqueous solubility of drugs is due to their hydrophobic core that provides a suitable microenvironment to accommodate hydrophobic substances (Alvarez-Lorenzo, Sosnik, Concheiro, 2011). In this way, the solubility of resveratrol in F127 micellar solutions $(5000 \mu \mathrm{M})$ was about 30 times higher than its solubility in pure water, which is about $131 \mu \mathrm{M}$ (Liu, Jiang, Han, 2012), probably due to the incorporation of the drug into the core of the micelles.

The mean diameter, zeta potential and $\mathrm{pH}$ value of each formulation are summarized in Table I and the size distribution is shown in Figure 1. The size of micelles is a very important parameter for drug delivery efficacy. The size variation, inside the nanoscale range, affects the blood circulation time and the bioavailability of the encapsulated drug (Kabanov et al., 2002). Considering several variants, such as renal clearance, penetration of small capillaries, capture by the phagocyte system and circulation time, the preferred size range for drug delivery using nanoscale particles is from 10 to $100 \mathrm{~nm}$ (Kabanov, Alakhov, 2002), as with those observed for the formulations MS-5+RES-2 and MS-10+RES-3. Furthermore, all formulations containing resveratrol could be classified as monodisperse (PI $\leq 0.3$ ), while the formulations without resveratrol were polidisperse (PI > 0.3) (Gaumet et al., 2008).
The micelles containing resveratrol exhibited a larger size than the micelles without resveratrol, indicative that the F127 micelles encapsulated the compound. Interestingly, the size increase was not directly proportional to the amount of resveratrol added, that is, MS-10+RES- 1 containing $500 \mu \mathrm{M}$ of resveratrol is larger than MS-5+RES-2 and MS-10+RES-3 containing $5000 \mu \mathrm{M}$ of resveratrol. We suggest that this effect occurred due to the aggregation of two or more micelles, since the formulation MS-10+RES-1 showed the lowest value, per module, of zeta potential (Varshosaz et al., 2018; Varshosaz et al., 2014) and greater zeta potential values ( $>30 \mathrm{mV}$, per module) are required to stabilize nanoparticles effectively by electric repulsion in liquid medium (Clogston, Patri, 2011).

Although the long-term stability of the micelles has not been evaluated, we suggest that they can be lyophilized to maintain their characteristics. Pepi et al. (2014) showed that the lyophilizing process of Pluronic ${ }^{\circledR}$ F127/L121 mixed micelles resulted in a reconstituted product with mostly similar hydrodynamic diameter and polydispersity index values to the fresh micelle formulation (Pepić et al., 2014).

Table II shows the results of resveratrol quantified in the formulations. The formulations showed between 95.9 and $96.8 \%$ encapsulation efficiency. Li et al. also showed high encapsulation rates when preparing mixed micelles (F127 and another Pluronic ${ }^{\mathbb{R}}$ ) containing resveratrol using the thin-film hydration method (Li et al., 2015). It was not possible to determinate the encapsulation efficiency of MS-5+RES-2 due to instability of the formulation one week after preparation.

TABLE I - Composition of micellar formulations, mean particle size, polydispersion index (PI), zeta potential and pH (mean \pm SD)

\begin{tabular}{lcccccc}
\hline Formulation & $\begin{array}{c}\text { RES concentration } \\
(\boldsymbol{\mu M})\end{array}$ & $\begin{array}{c}\text { \% } \\
\mathbf{F 1 2 7}\end{array}$ & $\begin{array}{c}\text { Mean particle size } \\
(\mathbf{n m})\end{array}$ & PI & $\begin{array}{c}\text { Zeta potencial } \\
(\mathbf{m V})\end{array}$ & pH \\
\hline MS-10+RES-1 & 500 & 10 & $209.70 \pm 81.71$ & $0.26 \pm 0.03$ & $-15.71 \pm 0.51$ & $6.59 \pm 0.03$ \\
MS-5+RES-2 & 5000 & 5 & $25.67 \pm 3.96$ & $0.30 \pm 0.03$ & $-22.80 \pm 6.68$ & $6.68 \pm 0.03$ \\
MS-10+RES-3 & 5000 & 10 & $28.32 \pm 3.24$ & $0.33 \pm 0.15$ & $-29.20 \pm 0.32$ & $6.62 \pm 0.05$ \\
MS-5 & - & 5 & $6.30 \pm 3.19$ & $0.69 \pm 0.38$ & $-12.30 \pm 6.17$ & $6.76 \pm 0.03$ \\
MS-10 & - & 10 & $5.30 \pm 0.07$ & $0.42 \pm 0.03$ & $-11.20 \pm 5.14$ & $6.76 \pm 0.02$ \\
\hline
\end{tabular}



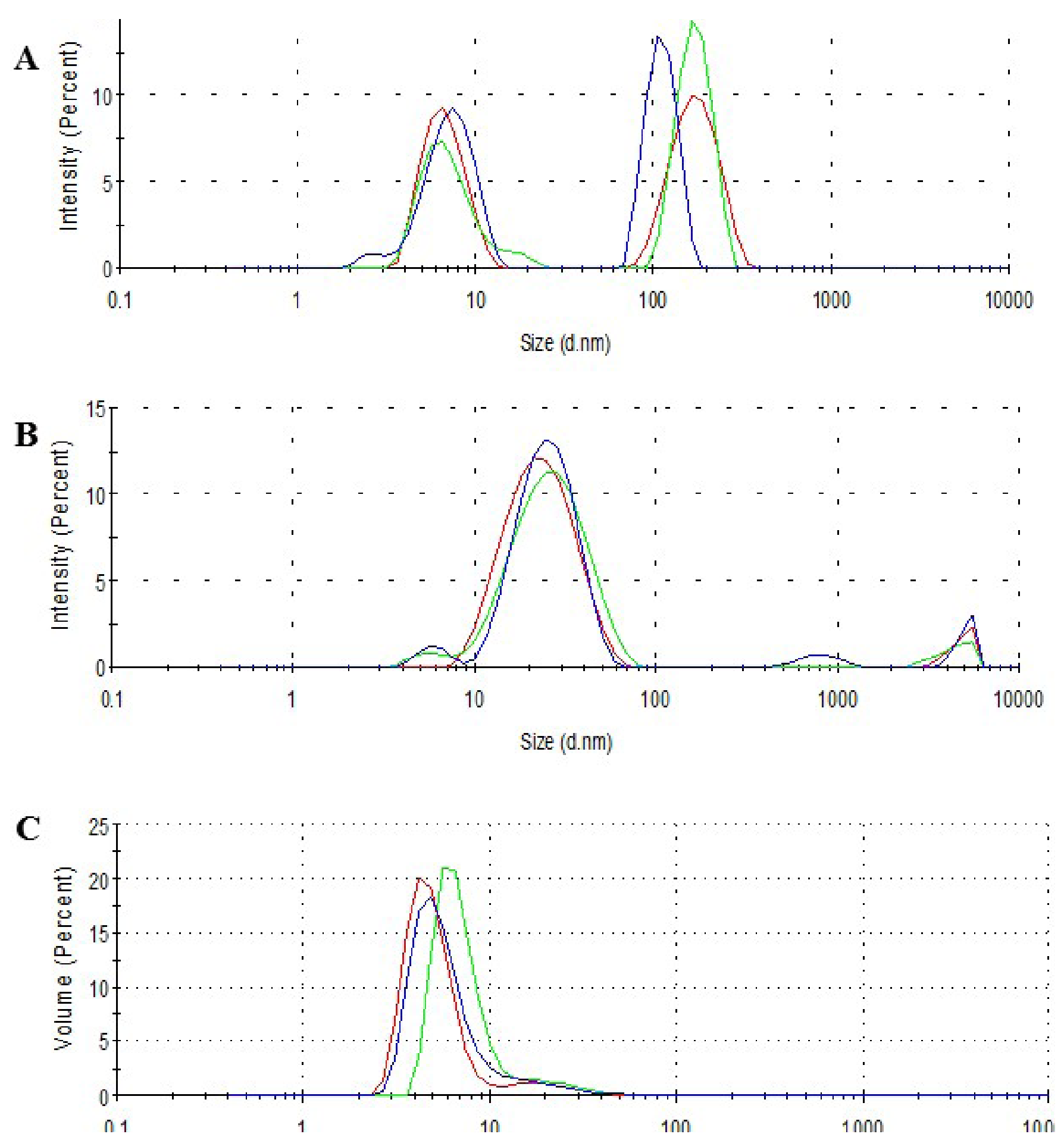

FIGURE 1 - Size distribution of micelles containing resveratrol. (A) MS-10+RES-1; (B) MS-5+RES-2; (C) MS-10+RES-3. 
TABLE II - Encapsulation efficiency

\begin{tabular}{lccccc}
\hline Formulation & $\begin{array}{c}\text { RES initial } \\
\text { concentration }(\boldsymbol{\mu M})\end{array}$ & $\begin{array}{c}\text { RES initial } \\
\text { mass }(\mathbf{m g})\end{array}$ & $\begin{array}{c}\text { RES final } \\
\text { concentration }(\boldsymbol{\mu M})\end{array}$ & $\begin{array}{c}\text { RES final } \\
\text { mass (mg) }\end{array}$ & $\begin{array}{c}\text { Encapsulation } \\
\text { efficiency }(\%)\end{array}$ \\
\hline MS-10+RES-1 & 500 & 1.41 & 484.10 & 1.36 & 96.82 \\
MS-5+RES-2 & 5000 & 11.41 & - & - & - \\
MS-10+RES-3 & 5000 & 11.41 & 4795.00 & 10.94 & 95.90 \\
\hline
\end{tabular}

RES: resveratrol; F127: Pluronic ${ }^{\circledR}$ F127; MS-10+RES-1: 10\% F127 and $500 \mu \mathrm{M}$ RES; MS-5+RES-2: 5\% F127 and $5000 \mu \mathrm{M}$ RES; MS-10+RES-3: 10\% F127 and $5000 \mu \mathrm{M}$ RES.

MS-10+RES-3 was chosen for evaluation of its antimicrobial and antitumoral activities, based on mean particle size, polydispersion index, zeta potential and encapsulation efficiency. Further characterization of the MS-10+RES-3 formulation was performed.

The results of micellar microenvironment analysis using fluorescence are shown in Figure 2. Changes in the microenvironment of micellar systems could affect the pyrene emission bands (Perry et al., 2011). The absorption bands of the MS-10 labeled with pyrene are very similar to the pyrene bands in methanol, showing the environment in which the pyrene is in micelles is more similar to methanol than to water. In this way, it can be confirmed that the probe has the ability to enter into the micelle. When the formulation MS-10+RES-3 was analyzed (Figure 2-B), a quenching effect was observed, that is, resveratrol promotes the decrease of pyrene fluorescence. Figure 2-B shows the gradual suppression of pyrene fluorescence in the presence of different concentrations of resveratrol. These data confirm that the F127 micelles encapsulated resveratrol.

While the F127 micellization process is strongly influenced by temperature (Dumortier et al., 2006), the in vitro release assay was conducted at $37{ }^{\circ} \mathrm{C}$, the same temperature at which the biological activities were carried out. As presented in Figure 3, there was a very low release of resveratrol from the formulation MS$10+$ RES-3. In 24 hours, only $0.45 \%$ of the resveratrol encapsulated in the micelles was released and in 48 hours, only $0.91 \%$. Although the in vitro release method used has already been described (Fonseca, 2016), it is possible that degradation of resveratrol occurs during the process due to $\mathrm{pH}$ (Zupancic, Lavric, Kristl, 2015). However, we suggest that the degradation was not the cause of the low concentration of resveratrol detected, since no different peaks were detected in the chromatogram. The release of hydrophobic drugs from structured nanoparticles in the form of core-corona is very dependent on the hydrophobic properties of the nucleus (Liu, Xiao, Allen, 2004). In the case of micelles formed by copolymers, the release rate is strongly influenced by the interaction forces occurring between the drug and the nucleus of the micelles (Allen et al., 2000). Therefore, the high encapsulation rates, which were achieved possibly by the strong interaction between resveratrol and the nucleus of F127 micelles, corroborates the low in vitro release for MS-10+RES-3 formulation.

The cytotoxic effects of MS-10+RES-3 on bladder cancer cells was evaluated. As presented in Figure 4, cytotoxicity was observed in the T24 cell line after treatment with MS-10+RES-3 and free resveratrol (150, 200 and $250 \mu \mathrm{M})$. Furthermore, the $\mathrm{IC}_{50}$ for MS-10+RES-3 (151.5 $\mu \mathrm{M})$ was lower than the $\mathrm{IC}_{50}$ for free resveratrol (178.73 $\mu \mathrm{M})$. Thus, we can affirm that the micellar formulation was able to preserve and improve the cytotoxic activity of resveratrol. Studies have showed that polymeric micelles based on Pluronics ${ }^{\circledR}$ can undergo internalization in cells and the general mechanism for absorption is endocytosis (Dehghankelishadi et al., 2017). On the other hand, some experiments indicate that encapsulated compounds may be released from the micelle and enter cells separately (Chen et al., 2008). Based on these findings and the in vitro release results, which showed low release of resveratrol during the same period used for the treatment of tumor cells, we suggest that the significant reduction of viability in the T24 cells was due to the internalization of the micelles by these cells. 
The results of antimicrobial activity are shown in Figure 5. For S. aureus, MS-10+RES-3 and free resveratrol were able to reduce the number of viable microorganisms at all tested concentrations, however, the activity of MS-10+RES-3 was higher than free resveratrol ( $\mathrm{p}<0.05$ for all tested concentrations). For
E. coli, MS-10+RES-3 and free resveratrol reduced the number of viable microorganisms at $62.5 \mu \mathrm{M}$ and $7.81 \mu \mathrm{M}$, respectively. For $C$. albicans, only the highest concentration of free resveratrol and MS$10+$ RES-3 $(250 \mu \mathrm{M})$ reduced the number of viable microorganisms.
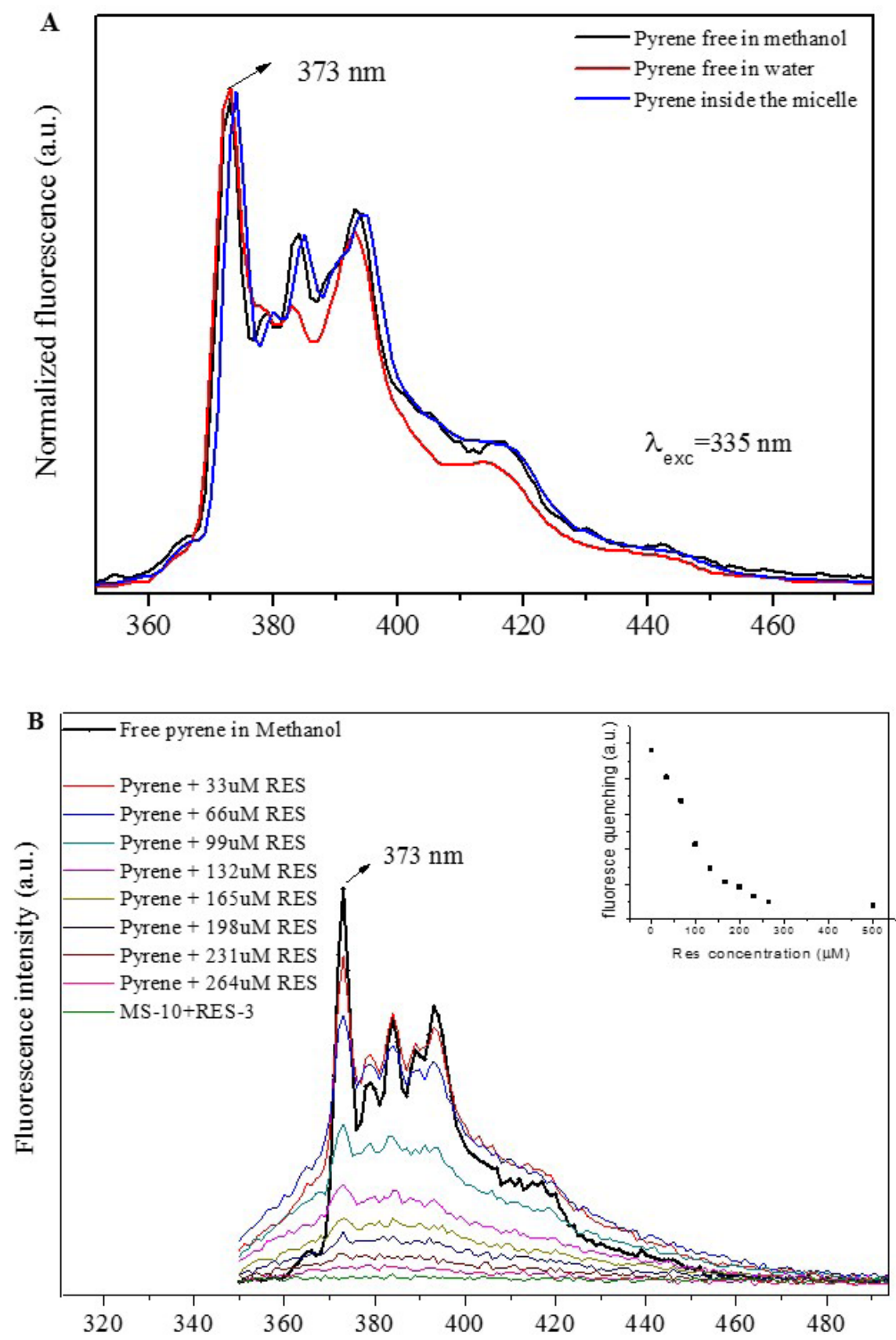

FIGURE 2 - Micellar microenvironment analysis using pyrene as a fluorescent probe. (A) Pyrene emission spectra in water, methanol and inside the micelles. (B) Pyrene emission spectra in presence of resveratrol and inside the formulation MS$10+$ RES-3. 


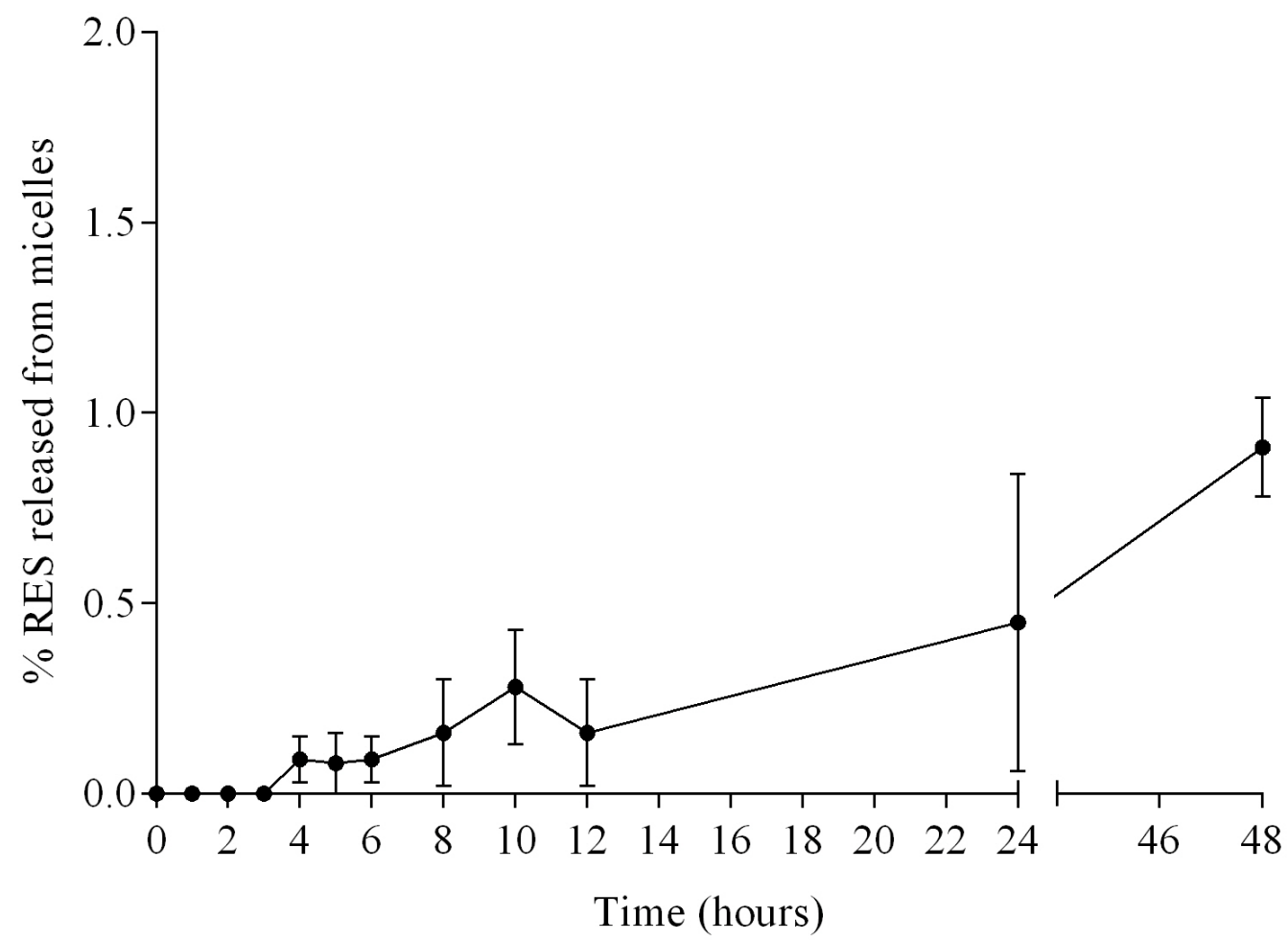

FIGURE 3 - Release of resveratrol from MS-10+RES-3 formulation. Each point represents the mean values \pm standard deviation obtained from three experiments.

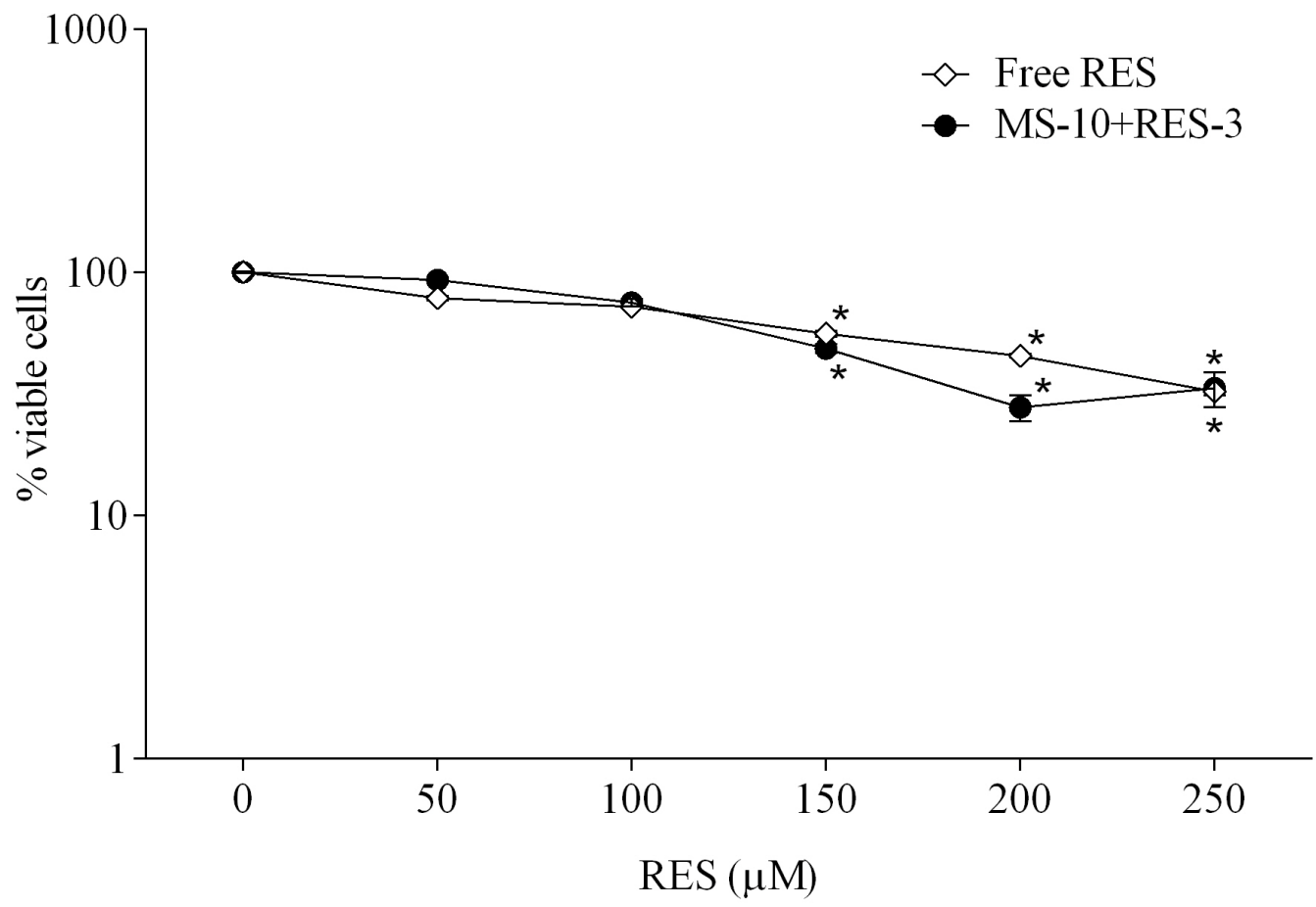

FIGURE 4 - Percentage of viable T24 cells (logarithmic scale) after treatment with free RES and formulation MS-10+RES-3 $(10 \% \mathrm{~F} 127$ and $5000 \mu \mathrm{M}){ }^{*} \mathrm{p}<0.05$ in relation to control without treatment with resveratrol. Each point represents the mean values \pm standard deviation obtained from three experiments. 

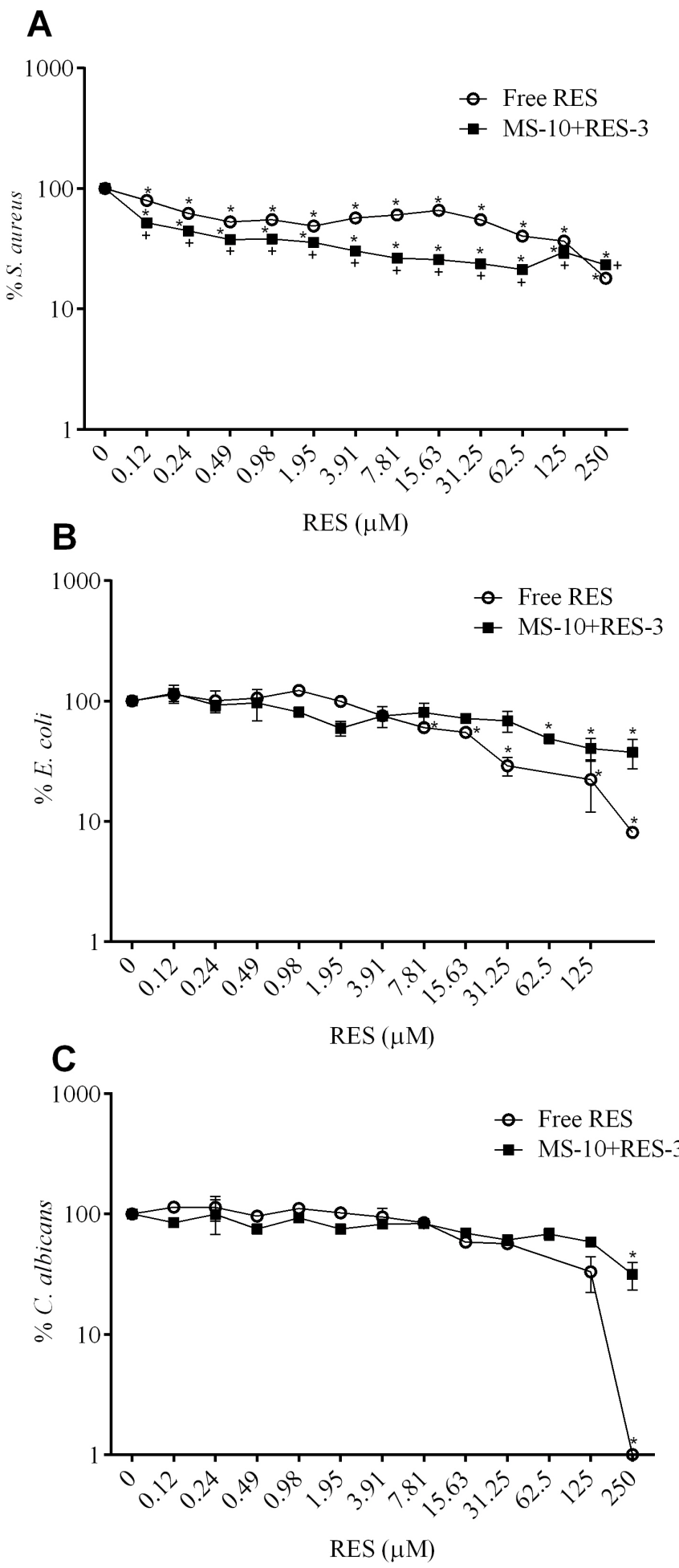

FIGURE 5 - Percentage of viable S. aureus (A), E. coli (B) and C. albicans (C) (logarithmic scale) after treatment with free resveratrol and formulation MS-10+RES-3 (10\% F127 and $5000 \mu \mathrm{M}) . * \mathrm{p}<0.05$ in relation to control without treatment with resveratrol. $+p<0.05$ in relation to free resveratrol. Each point represents the mean values \pm standard deviation obtained from three experiments.
Lower concentrations of free and formulated resveratrol inhibited viability of $S$. aureus $(0.12 \mu \mathrm{M})$ compared to E. coli ( $7.81 \mu \mathrm{M}$ and $62.5 \mu \mathrm{M}$, respectively) and $C$. albicans $(250 \mu \mathrm{M})$. Most of the cell wall of gram positive bacteria consists only of a peptidoglycan layer and it can be easily penetrated (Rajagopal, Walker, 2017). The cell wall structure of gram negative bacteria is characterized by an outer membrane constituted essentially of lipopolysaccharides, which confers increased resistance (Rojas et al., 2018). The main component of the cell wall of the yeast is chitin and ergosterol that modulate the fluidity of the membrane and prevent its alterations (Thevissen et al., 2003). Thus, generally, drugs have better effect against the gram positive bacteria.

The formulation MS-10+RES-3 showed a significant improvement in the antimicrobial activity of resveratrol against $S$. aureus, but not the other microorganisms tested, in which it was only able to retain the same activity. This finding could be due to differences in their cell membrane constituents and structure that provide different types of interaction of resveratrol compared to the formulation (Glisoni, Sosnik, 2014). Despite the fact that the MS-10+RES-3 formulation does not show a statistical increase of antimicrobial activity for all tested microorganisms, it is still an alternative for resveratrol use. Nanostructured systems provide antimicrobial advantages such as overcoming physical barriers and reaching difficult sites of action (Purro et al., 2018). In relation to its use in tumor cell treatment, besides the increase in cytotoxic activity, with reduction of the $\mathrm{IC}_{50}$, this kind of formulation is able to facilitate the delivery of drugs to cancer cells through the effect of increased permeability and retention (Biswas et al., 2016). Furthermore, some micellar formulations have already been moved to clinical trials (Lee et al., 2008; Valle et al., 2010), showing that they are potential formulations for clinical practice.

To our knowledge, this is the first study that evaluated the antimicrobial potential and cytotoxicity on bladder cancer cells of micellar formulations containing resveratrol, suggesting that converting this activity to nano-scale particles can feasibly improve its use, since we showed enhancement of the solubility of resveratrol and adequate parameters of size, PI and zeta potential. Furthermore, this study demonstrated the preservation of biological activities from resveratrol after its encapsulation in micellar systems. As suggested by other authors (Gu et al., 2017; Pellosi et al., 2016; Yu 
et al., 2015), our micellar formulation could be applied by intravenous or subcutaneous routes.

\section{CONCLUSION}

We used a simple and rapid method, without the use of organic solvents, for improving the solubility of resveratrol while maintaining its bacteriostatic and cytotoxic activities. These results showed that micellar nanostructures could ensure the maintenance of the biological activity of resveratrol, besides favoring its applicability in biological systems and clinical practices.

\section{ACKNOWLEDGEMENTS}

This study was supported by Fundação de Amparo à Pesquisa do Estado de Minas Gerais (CBBAPQ-01497-14 and PPM-00282-11), Conselho Nacional de Desenvolvimento Científico e Tecnológico (CNPq441836/2014-3) and Universidade Federal de Ouro Preto (23109.003517/2018-85). Thanks to Global Science Editing Ltd. for English language correction.

\section{REFERENCES}

Akash MS, Rehman K. Recent progress in biomedical applications of Pluronic (PF127): Pharmaceutical perspectives. J Control Release. 2015;209:120-38.

Allen C, Han J, Yu Y, Maysinger D, Eisenberg A. Polycaprolactone-b-poly (ethylene oxide) copolymer micelles as a delivery vehicle for dihydrotestosterone. J Control Release. 2000;63(3):275-286.

Alvarez-Lorenzo C, Sosnik A, Concheiro A. PEO-PPO block copolymers for passive micellar targeting and overcoming multidrug resistance in cancer therapy. Curr Drug Targets. 2011;12(8):1112-1130.

Amri A, Chaumeil JC, Sfar S, Charrueau C. Administration of resveratrol: What formulation solutions to bioavailability limitations? J Control Release. 2012;158(2):182-193.

Bai Y, Mao QQ, Qin J, Zheng XY, Wang YB, Yang K, et al. Resveratrol induces apoptosis and cell cycle arrest of human T24 bladder cancer cells in vitro and inhibits tumor growth in vivo. Cancer Sci. 2009;101(2):488-93.

Biswas S, Kumari P, Lakhani PM, Ghosh B. Recent advances in polymeric micelles for anti-cancer drug delivery. Eur J Pharm Sci. 2016;83:184-202.
Bodratti AM, Alexandridis P. Formulation of poloxamers for drug delivery. J Funct Biomater. 2018;9(1):1-24.

Chen H, Kim S, Li L, Wang S, Park K, Cheng J. Release of hydrophobic molecules from polymer micelles into cell membranes revealed by Förster resonance energy transfer imaging. Proc Natl Acad Sci-Biol. 2008;105(18):6596-6601.

Clinical and Laboratory Standards Institute (CLSI). Methods for Dilution Antimicrobial Susceptibility Tests for Bacteria That Grow Aerobically; Approved Standard. $2^{\text {th }}$ ed. 2012;32:1-88.

Clogston JD, Patri AK. Zeta potential measurement. In: Characterization of nanoparticles intended for drug delivery. Human Press; 2011; p.63-70.

Dehghankelishadi P, Saadat E, Ravar F, Safavi M, Pordeli M, Gholami M, et al. In vitro and in vivo evaluation of paclitaxellapatinib-loaded F127 pluronic micelles. Drug Dev Ind Pharm. 2017;43(3):390-398.

Dumortier G, Grossiord JL, Agnely F, Chaumeil JC. A review of poloxamer 407 pharmaceutical and pharmacological characteristics. Pharm Res. 2006;23(12):2709-2728.

Euba B, López-López N, Rodríguez-Arce I, Fernández-Calvet A, Barberán M, Caturla N, et al. Resveratrol therapeutics combines both antimicrobial and immunomodulatory properties against respiratory infection by nontypeable Haemophilus influenzae. Sci Rep. 2017;7:12860.

Figueiró F, Bernardi A, Frozza RL, Terroso T, ZanottoFilho A, Jandrey EH, et al. Resveratrol-loaded lipid-core nanocapsules treatment reduces in vitro and in vivo glioma growth. J Biomed Nanotechnol. 2013;9(3):516-526.

Fonseca DP. Obtenção, caracterização e avaliação das atividades antioxidante e antitumoral in vitro de nanopartículas de albumina contendo resveratrol [dissertation]. Universidade Estadual do Centro-Oeste; 2016.

Francioso A, Mastromarino P, Masci A, d'Erme M, Mosca L. Chemistry, stability and bioavailability of resveratrol. Med Chem. 2014;10(3):237-245.

Friedrich RB, Kann B, Coradini K, Offerhaus HL, Beck RC, Windbergs M. Skin penetration behavior of lipid-core nanocapsules for simultaneous delivery of resveratrol and curcumin. Eur J Pharm Sci. 2015;12:204-213.

Gaumet M, Vargas A, Gurny R, Delie F. Nanoparticles for drug delivery: the need for precision in reporting particle size parameters. Eur J Pharm Biopharm. 2008;69(1):1-9. 
Giuliano E, Paolino D, Fresta M, Cosco D. Mucosal applications of poloxamer 407-based hydrogels: an overview. Pharmaceutics. 2018;10(3):pii:E159.

Glisoni RJ, Sosnik A. Encapsulation of the antimicrobial and immunomodulator agent nitazoxanide within polymeric micelles. J Nanosci Nanotechnol. 2014;14(6):4670-82.

Gu W, Chen J, Patra P, Yang X, Gu Q, Wei L, et al. Nanoformulated water-soluble paclitaxel to enhance drug efficacy and reduce hemolysis side effect. J Biomater Appl. 2017;32(1):66-73.

Hao J, Tong T, Jin K, Zhuang Q, Han T, Bi Y, et al. Folic acid-functionalized drug delivery platform of resveratrol based on Pluronic 127/D- $\alpha$-tocopheryl polyethylene glycol 1000 succinate mixed micelles. Int J Nanomedicine. 2017;12:2279-2292.

Hussein YHA, Youssry M. Polymeric Micelles of biodegradable diblock copolymers: enhanced encapsulation of hydrophobic drugs. Materials. 2018;11(5):E688.

Hwang D, Lim Y. Resveratrol antibacterial activity against Escherichia coli is mediated by Z-ring formation inhibition via suppression of FtsZ expression. Sci Rep. 2015;5:10029.

Ji Q, Liu X, Han Z, Zhou L, Sui H, Yan L, et al. Resveratrol suppresses epithelial-to-mesenchymal transition in colorectal cancer through TGF- $\beta 1 /$ Smads signaling pathway mediated Snail/E-cadherin expression. BMC Cancer. 2015;15:97.

Kabanov AV, Alakhov VY. Pluronic ${ }^{\circledR}$ block copolymers in drug delivery: from micellar nanocontainers to biological response modifiers. Crit Rev Ther Drug. 2002;19(1):1-73.

Kabanov AV, Batrakova EV, Alakhov VY. Pluronic block copolymers as novel polymer therapeutics for drug and gene delivery. J Control Release. 2002;82(2-3):189-212.

Kumar A, Rimando AM, Levenson AS. Resveratrol and pterostilbene as a microRNA-mediated chemopreventive and therapeutic strategy in prostate cancer. Ann N Y Acad Sci. 2017;1403(1):15-26.

Lee J, Lee DG. Novel antifungal mechanism of resveratrol: apoptosis inducer in Candida albicans. Curr Microbiol. 2015;70(3):383-389.

Lee K, Lee JH, Ryu SY, Cho MH, Lee J. Stilbenes reduce Staphylococcus aureus hemolysis, biofilm formation and virulence. Foodborne Pathog Dis. 2014;11(9):710-717.

Lee KS, Chung HC, Im SA, Park YH, Kim CS, Kim SB, et al. Multicenter phase II trial of Genexol-PM, a Cremophorfree, polymeric micelle formulation of paclitaxel, in patients with metastatic breast cancer. Breast Cancer Res Tr. 2008;108(2):241-250.

Li JF, Gao MY, Wang HM, Liu QY, Mao SR. Optimization and in vitro characterization of resveratrol-loaded poloxamer 403/407 mixed micelles. Yao Xue Xue Bao. 2015;50(8): 1045-1051.

Liu J, Xiao Y, Allen C. Polymer-drug compatibility: a guide to the development of delivery systems for the anticancer agent, ellipticine. J Pharm Sci. 2004;93(1):132-43.

Liu X, Jiang Z, Han J. Increasing solubility of poorly water soluble drug resveratrol by surfactants and cyclodextrins. Adv Mat Res. 2012;418-420:2231-2234.

Liu Y, Fu S, Lin L, Cao Y, Xie X, Yu H, et al. Redoxsensitive Pluronic F127-tocopherol micelles: synthesis, characterization, and cytotoxicity evaluation. Int $\mathrm{J}$ Nanomedicine. 2017;12:2635-2644.

Nie P, Hu W, Zhang T, Yang Y, Hou B, Zou Z. Synergistic induction of erlotinib-mediated apoptosis by resveratrol in human non-small-cell lung cancer cells by down-regulating survivin and up-regulating PUMA. Cell Physiol Biochem. 2015;35(6):2255-2257.

Oerlemans C, Bult W, Bos M, Storm G, Nijsen JF, Hennink WE. Polymeric micelles in anticancer therapy: targeting, imaging and triggered release. Pharm Res. 2010;27(12):2569-2589.

Owen SC, Chan DPY, Shoichet MS. Polymeric micelle stability. Nano Today. 2012;7(1):53-65.

Patel KR, Brown VA, Jones DJ, Britton RG, Hemingway D, Miller AS, et al. Clinical pharmacology of resveratrol and its metabolites in colorectal cancer patients. Cancer Res. 2010;70(19):7392-7399.

Pellosi DS, Moret F, Fraix A, Marino N, Maiolino S, Gaio E, et al. Pluronic ${ }^{\circledR}$ P123/F127 mixed micelles delivering sorafenib and its combination with verteporfin in cancer cells. Int J Nanomedicine. 2016;11:4479-4494.

Pepić I, Lovrić J, Hafner A, Filipović-Grčić J. Powder form and stability of Pluronic mixed micelle dispersions for drug delivery applications. Drug Dev Ind Pharm. 2014;40(7): 944-951.

Perry CC, Sabir TS, Livingston WJ, Milligan JR, Chen Q, Maskiewicz V, et al. Fluorescence of commercial Pluronic F127 samples: Temperature-dependent micellization. J Colloid Interface Sci. 2011;354(2):662-669.

Purro M, Qiao J, Liu Z, Ashcraft M, Xiong MP. Desferrioxamine:gallium-pluronic micelles increase outer 
membrane permeability and potentiate antibiotic activity against Pseudomonas aeruginosa. Chem Commum (Camb). 2018;54(99):13929-13932.

Rajagopal M, Walker S. Envelope structures of Gram-positive bacteria. Curr Topics Microbiol Immunol. 2017;404:1-44.

Rey-Rico A, Cucchiarini M. PEO-PPO-PEO tri-block copolymers for gene delivery applications in human regenerative medicine-an overview. Int $\mathrm{J}$ Mol Sci. 2018;19(3):pii:E775.

Rijcken CJ, Hofman JW, van Zeeland F, Hennink WE, van Nostrum CF. Photosensitiser-loaded biodegradable polymeric micelles: preparation, characterization and in vitro PDT efficacy. J Control Release. 2007;124(3):144-153.

Rojas ER, Billings G, Odermatt PD, Auer GK, Zhu L, Miguel $\mathrm{A}$, et al. The outer membrane is an essential load-bearing element in Gram-negative bacteria. Nature. 2018;559:617-621.

Santos AC, Veiga F, Ribeiro AJ. New delivery systems to improve the bioavailability of resveratrol. Expert Opin Drug Deliv. 2011;8(8):973-990.

Selvaraj S, Sun Y, Sukumaran P, Singh BB. Resveratrol activates autophagic cell death in prostate cancer cells via downregulation of STIM1 and the mTOR pathway. Mol Carcinog. 2016;55(5):818-831.

Stammet M; Kwon GS, Rao DA. Drug loading in Pluronic ${ }^{\circledR}$ micelles made by solvent casting and equilibrium methods using resveratrol as a model drug. J Control Release. 2010;148(1):21-56.

Taylor EJM, Yu Y, Champer J, Kim, J. Resveratrol demonstrates antimicrobial effects against Propionibacterium acnes in vitro. Dermatol Ther. 2014;4(2):249-257.

Thevissen K, Ferket KKA, François IEJA, Cammue BPA. Interactions of antifungal plant defensins with fungal membrane components. Peptides. 2003;24(11):1705-1712.

Trela BC, Waterhouse AL. Resveratrol: isomeric molar absorptivities and stability. J Agric Food Chem. 1996;44(5):1253-1257.
Valle J, Armstrong A, Newman C, Alakhov V, Pietrzynski G, Brewer J, et al. A phase II study of SP1049C, doxorubicin in P-glycoprotein-targeting pluronics, in patients with advanced adenocarcinoma of the esophagus and gastroesophageal junction. Invest New Drugs. 2010;29(5):1029-1037.

Varshosaz J, Hassanzadeh F, Sadeghi-Aliabadi H, Firozian F. Uptake of etoposide in CT-26 cells of colorectal cancer using folate targeted dextran stearate polymeric micelles. Biomed Res Int. 2014;2014:708593.

Varshosaz J, Taymouri S, Minaiyan M, Rastegarnasab F, Baradaran A. Development and in vitro/in vivo evaluation of HPMC/chitosan gel containing simvastatin loaded selfassembled nanomicelles as a potent wound healing agent. Drug Dev Ind Pharm. 2018;44(2):276-288.

Vitonyte J, Manca ML, Caddeo C, Valenti D, Peris JE, Usach I, et al. Bifunctional viscous nanovesicles co-loaded with resveratrol and gallic acid for skin protection against microbial and oxidative injuries. Eur J Pharm Biopharm. 2017;114:278-287.

Wang DG, Liu WY, Chen GT. A simple method for the isolation and purification of resveratrol from Polygonum cuspidatum. J Pharm Analysis. 2013;3(4):241-247.

Wang J, Liu Q, Yang L, Xia X, Zhu R, Chen S, et al. Curcuminloaded TPGS/F127/P123 mixed polymeric micelles for cervical cancer therapy: formulation, characterization, and in vitro and in vivo evaluation. J Biomed Nanotechnol. 2017;13(12):1631-46.

Wu ML, Li H, Yu LJ, Chen XY, Kong QY, Song X, et al. Short-term resveratrol exposure causes in vitro and in vivo growth inhibition and apoptosis of bladder cancer cells. PLoS One. 2014;9(2):89806.

Yu ZG, Geng ZX, Liu TF, Jiang F. In vitro and in vivo evaluation of an in situ forming gel system for sustained delivery of Florfenicol. J Vet Pharmacol Ther. 2015;38(3): 271-277.

Zupancic S, Lavric Z, Kristl J. Stability and solubility of transresveratrol are strongly influenced by $\mathrm{pH}$ and temperature. Eur J Pharm Biopharm. 2015;93:196-204.

Received for publication on $05^{\text {th }}$ June 2018 Accepted for publication on $08^{\text {th }}$ January 2019 